\title{
Mechanical and Control System Digital Simulation Based on MATLAB
}

\author{
Caixia Han \\ School of Information technology, College of science \& Arts of Jianghan University. China
}

\begin{abstract}
With the rapid development of power electronic technology, microelectronics technology, modern control theory and material science, AC speed control system and electromechanical integration technology presents a broad development and application prospect. Therefore, it is has become a challenging new topic to set up models, make simulation and analysis for such system. In this paper, it takes the simulation of mechanical control system as the breakthrough point, combined with the brief introduction of Matlab/Simulink simulation software and the general method for simulation of mechanical and control system, discussing the realization of the general simulation model of the mechanical and control system based on MATLAB/Simulink.
\end{abstract}

Keywords: Matlab/Simulink software; Mechanical control system; Digital simulation

\section{Introduction}

Mechanical engineering control takes the generalized system dynamics related to mechanical engineering as the researching object, so as to go on with the transmission, processing and feedback of the system. As for high order systems, the time response curves and frequency characteristics of Bode, Nyquist, Nichols, and so on, it is difficult to accurately draw, which is difficult to analyze the characteristics of the system intuitively.[1] At present, because MATLAB/Simulink simulation platform has been widely recognized by the scientific and technological community because for its advantages such as convenient operation, rich model base and high efficiency of simulation and so on, which has become the first choice for simulation of the system. In this paper, it takes MATLAB as a tool, discussing the general rule of the digital simulation of mechanical and control system based on MATLAB.

\section{The Interpretation of Simulation of Mechanical Control System}

Simulation can use the physical or mathematical model to simulate the process of the reality, so as to seek the understanding of the real process. The basic principle that it follows is the principle of similarity. While computer simulation is based on the simulation model of the system, which is the method of analysis and research by using computer. At present, the general used computer simulation software can be including Proteus, LABVIEW, ORCAD, SYSTEMVIEW, MATLAB, SIMULINK and so on.[2] MATLAB is a kind of advanced scientific analysis and computing software. It has powerful numerical calculation ability and all kinds of tools, which has the advantages of object-oriented and visualization, while SIMULINK is a dynamic simulation environment based on the model of MATLAB. At present, MATLAB and SIMULINK are the digital simulation software for the control system that are widely adopted by people, while Graphical User Interface (GUI) is the graphical interface development environment of MATLAB, which can construct simulation system out from the MATLAB environment.

\section{Brief Introduction about Matlab/Simulink Software}

MATLAB language is an engineering application software which can integrate scientific computing, data visualization and program design. It has become the essential basic software for the subject of computer to have aided engineering analysis, design, simulation as well as teaching, which is composed of MATLAB main package, Simulink module and toolbox with various functions.[3] Among the, the toolbox of the control system can provide a wealth of functions for processing and analyzing LTI model, which can not only realize the establishment of system model, as well as the conversion, analysis and processing of the system, which also can design the control system, thus, it 
is convenient for mechanical engineering control system to have simulation on time response analysis, as well as frequency characteristics analysis. As an important component of Simulink, MATLAB module can support linear and nonlinear systems, continuous and discrete time systems, continuous and discrete hybrid systems, which has become the most widely used simulation software for dynamic system.Simulink can provide a friendly graphical user interface, the simulation model is connected to a block diagram by a series of modules, which can complete the task through a simple click and the dragging function of the mouse. The module library of Simulink consists of system modules such as:continuous system, discrete system, nonlinear system and so on, which also consists of function and form module group, math module group, signal and system module group, signal source module group and output module group.After the simulation model is established, the appropriate signal source module can be chosen as the input signal, observing the response of the system with the appropriate output module, then the system characteristics can be analyzed.[4]

\section{General Method for Simulation of Inspection and Control System}

The general process of simulation of inspection and control system can be including three stages: the establishment of mathematical model, the establishment of the simulation model (also known as the two modeling) and the simulation experiment. In this paper, it mainly discusses the method of realizing the mathematical model to the simulation model and the realization method of the simulation model on the MATLAB/Simulink platform. Moreover, the simulation algorithm is also described in this part. Finally, the evaluation principle of the simulation results as well as the standardization of data processing method can also be given, which can play a certain significant role in guiding the digital simulation for inspection and control system.

\section{System Modeling and Model Transformation}

\subsection{The Overview of System Modeling}

Modeling is the abstract mathematical model of motion law based on the basic physical law of the researching object, so as to describe the process of modeling. Generally speaking, there are two ways of modeling for the system. One is the mechanism analysis, which is based on the mechanism of the system (such as physical and chemical laws), so as to establish the system model, thus, it is usually called "white box" problem. Inspection and control system generally has the certain physical structure and electromagnetic relationship determined by the general modeling with mechanism analysis; the other is the experimental method, using the input and output signal of the system to deduce the mathematical model of the system, which can be known as the "black box". The experimental method is generally used in modeling with the uncertain model (such as sociology, statistics, etc.), sometimes it also can be used in the inspection and control system model, such as the magnetization curve obtained by the experiment that can be corrected by the motor's mathematical model, so that the model can be more accurate.

\subsection{General Method of Simulation Model}

After the mathematical model of inspection and control system is determined, it needs to transform it into simulation model that computer can make calculation, which also can be known as the second modeling. Generally speaking, the mathematical model is needed to be transformed into the standard form of the state equation, and the initial conditions of the state variables should be determined.

\subsection{The Conversion from Standard State Equation to Differential Equations}

Generally, the inspection and control system can be described by differential equation, it should select the status variable firstly, when it is transformed into the standard state equation. When it takes the saturation of magnetic field into consideration, the flux linkage can be selected as the state variable, and the inductance can be modified according to the magnetization curve during this process, otherwise it can select the current state variable, which can make it more intuitive and convenient [5].

\subsection{The Processing Method of High Order Ordinary Differential Equation}

Assuming the general form of the higher order ordinary differential equation is: 


$$
y^{(n)}=f\left(t, y, \bar{y}, \ldots \ldots, y^{(n-1)}\right)
$$

Moreover,the initial value of the $y(t)$ is known with the initial value of the output variable $y(0), \bar{y}(0), \ldots \ldots, y^{(n-1)}(0)$, then it can select a group of state variable $x_{1}=y, x_{2}=\bar{y}, \ldots \ldots, x_{n}=y^{(n-1)}$, therefore, it can transform the original high order differential equation model into the equivalent first-order differential equations, i.e.

$$
\left\{\begin{array}{c}
\bar{x}_{1}=x_{2} \\
\bar{x}_{2}=x_{3} \\
\vdots \\
\bar{x}_{n}=f\left(t, x_{1}, x_{2}, \cdots, x_{n}\right)
\end{array}\right.
$$

And the initial value $x_{1}(0)=y(0), x_{2}(0)=\bar{y}(0), \ldots \ldots, x_{n}(0)=y^{(n-1)}(0)$.

\subsection{The Realization of General Simulation Model of Inspection and Control System Based on MATLAB/Simulink}

Mathematical model of electromechanical dynamic system ultimately can be converted into standard form of equation of state, which can make these mathematical expression be accepted by the computer, so as to solve description problem of the equations of state on the Matlab/Simulink platform ultimately.Electromechanical dynamic simulation model typically can be including the following three types of elements: input module, simulation module of electrical and mechanical dynamic system, as well as the output displaying module. In general, the terminal interface of the inspection and control system's constraint can be regarded as the part of the input part of the model, such as terminal voltage, load torque and so on. Taking the equation of state as part of the core module of the system, sending the evaluated state or the result calculated by the state variable to the output displaying module, such as current, flux, electromagnetic torque and so on.

\subsection{Modeling of Using $S$ - function Method}

S-function is the short term for the function of system, it can adopt a non graphical way to describe a module. All of the function modules in Simulink can be described by $\mathrm{S}$ function. $\mathrm{S}$ function can support MATLAB, C, C++, Fortran or Ada language, S-function that used M language to write can call both toolbox and graphics function, which also can use pure mathematic expression to write the equation of state, which is very suitable for the description of complicated system, moreover, it can provide users with a better platform with autonomous development.

Table 1 Contents of S- function

\begin{tabular}{ccc}
\hline Simulation stage & Order of S- function & flag \\
\hline $\begin{array}{c}\text { Initialization, defining the sampling time,the initial condition and } \\
\text { the sizes array of the continuous state, and the discrete state }\end{array}$ & mdl Initialized Sizes & flag=0 \\
Calculating the next sampling time & mdl Get Time Of Next Var Hit & flag=4 \\
Given $t, \mathrm{x}, \mathrm{u}$, calculating the output of S- function & mdl Outputs & flag=3 \\
Updating the discrete state, sampling time and maximum time step & mdl Update & flag=2 \\
Given t, $\mathrm{x}, \mathrm{u}$, calcualting the derivative number of the continuous & mdl Derivatives & flag=1 \\
state & mdl Terminate & flag=9 \\
\hline
\end{tabular}

\section{Conclusion}

MATLAB/Simulink simulation platform can have powerful graphics capabilities and data processing capabilities, based on the Simulink platform, the simulation results of mechanical and electrical power system can use the oscilloscope Scope module to observe, which also can use the display module to display the real-time data. Generally, it can use the oscilloscope Scope module to have observation. From the above we can see that digital simulation is very necessary for the analysis and design of the system. It can deepen the understanding of the testing circuit as well as the working principle of the system, accelerating the design and theory of the system, so as to help researchers improve their own level of development, improve product performance that can shorten the renewal cycle of products effectively. 


\section{References}

[1]. S.Peresada. 1999. High-performance indirect field-oriented output-feedback control of induction motors. Automatic. vol.35, pp: 1033-1047.

[2]. Cho Soon-Bong, Myoung-Ho Shin, Hyun Dong-Seok. 2000. An improved stator flux estimation for speed sensorless stator flux orientation control of induction motors. IEEE Trans. POW. Electron. vol.15, pp: 312-317.

[3]. Sul Seung Ki, Kim.Jang Hwan, Choi. Jong Woo. 2002. Novel Rotor-Flux observer using observer characteristic function in Complex vector space for field-oriented induction motor drives. IEEE Trans I. A. vol.38, pp: 1334-1343.

[4]. Hoang Le Huy, G.Sybille. 2000. MATLAB/Simulink and PSPice as modelling tools for power systems and power electronics.

[5]. Qunjing wang, Tao Zhao. 2005. Application of MATLAB/SIMULINK and PSPICE simulation in teaching power electronics and electric drive system. 